\title{
Norois
}

Environnement, aménagement, société

$243 \mid 2017$

Capital environnemental et espaces emblématiques

\section{Le capital environnemental, nouvelle clé d'interprétation de la gentrification rurale?}

Environmental capital, a new way of interpreting rural gentrification?

Frédéric Richard, Greta Tommasi et Gabrielle Saumon

\section{(2) OpenEdition}

Édition électronique

URL : http://journals.openedition.org/norois/6106

DOI : 10.4000/norois.6106

ISBN : 978-2-7535-6537-7

ISSN : 1760-8546

Éditeur

Presses universitaires de Rennes

Édition imprimée

Date de publication : 2 novembre 2017

Pagination : 89-110

ISBN : 978-2-7535-6465-7

ISSN : 0029-182X

\section{Référence électronique}

Frédéric Richard, Greta Tommasi et Gabrielle Saumon, « Le capital environnemental, nouvelle clé d'interprétation de la gentrification rurale? », Norois [En ligne], 243 | 2017, mis en ligne le 20 septembre 2019, consulté le 15 mars 2021. URL : http://journals.openedition.org/norois/6106 ; DOI : https:// doi.org/10.4000/norois.6106

(c) Tous droits réservés 


Presses
Universitaires
de Rennes
www.pur-editions.fr

\title{
Le capital environnemental, nouvelle clé d'interprétation de la gentrification rurale?
}

\author{
Environmental Capital, a New Way of Interpreting Rural Gentrification?
}

Frédéric RicharD ${ }^{* a}$, Greta Tommasi ${ }^{a}$ et Gabrielle SAumon ${ }^{a}$

\footnotetext{
* Auteur correspondant

a GEOLAB - UMR 6042 CNRS, université de Limoges, faculté des lettres et des sciences humaines - 39E rue Camille-Guérin,87036 Limoges, France.(frederic.richard@unilim.fr; greta.tommasi@unilim.fr; gabrielle.saumon@ unilim.fr)
}

Résumé : Encore confidentielle en France, mais déjà bien ancrée dans la littérature anglo-saxonne, l'approche proposée par la gentrification rurale permet une lecture renouvelée des dynamiques contemporaines des espaces ruraux, mettant entre autres choses l'environnement au cœur des transformations socio-spatiales et soulignant les inégalités liées à ce processus. À travers les recherches empiriques menées dans trois terrains singuliers mais unis par leur caractère emblématique (les campagnes anglaises, le Montana, la Montagne limousine), l'article propose d'explorer le processus de gentrification en l'interrogeant par le prisme du capital environnemental : cet actif social rend compte des investissements des gentrifieurs dans l'environnement, réalisés selon des valeurs spécifiques, dans le but de satisfaire leurs intérêts et leurs objectifs. Par le jeu de conversion et reconversion des capitaux qu'elle suggère, cette grille de lecture permet alors d'appréhender les rapports de force et les inégalités d'accès à l'environnement que le processus implique, et se révèle un outil efficace pour une lecture transnationale de la gentrification rurale.

\begin{abstract}
Rural gentrification - as yet a little-known phenomenon in France but one that is well established in the Anglo-Saxon academic literature - offers a new standpoint from which to analyze the contemporary dynamics of rural spaces. Among other things, it puts environmental issues at the heart of sociospatial changes and underlines the social inequalities linked to this process. Through three empirical studies realized in three quite different areas, but which all benefit from emblematic identities (the English countryside, Montana in the US and the Montagne Limousine in France), this paper seeks to investigate the gentrification process through the lens of environmental capital. This social asset can be defined as degree which gentrifiers invest in the environment, in accordance with specific values, with the aim of achieving their own goals and satisfying their own interests. As a result of the conversion and re-conversion of different kinds of assets that this suggests, this analysis framework helps us to understand the balance of power at play and the inequalities that exist in terms of access to the environment, and reveals itself to be an efficient tool for the transnational analysis of rural gentrification.
\end{abstract}

Mots clés : Capital environnemental - gentrification rurale - recomposition sociale - inégalités - domination - conflit environnemental

Keywords: Environmental capital - rural gentrification - social change - inequalities - domination - environmental conflict 


\section{INTRODUCTION}

Si elle est encore restée relativement confidentielle en France, l'approche des mutations rurales par le prisme conceptuel de la gentrification est déjà bien ancrée dans le paysage académique anglo-saxon. De manière générique, ce processus décrit l'installation sur un territoire de nouveaux habitants mieux pourvus que les populations antérieurement implantées en capital économique, mais également culturel et/ou social. Se traduisant par l'exclusion plus ou moins marquée des ménages les plus modestes, ce mouvement s'accompagne de profondes recompositions socio-économiques et paysagères des campagnes, ainsi que des représentations dont elles font l'objet. À leurs débuts, les travaux sur la gentrification rurale ont surtout mis l'accent sur l'articulation entre les politiques de planification (zonages, contrôle strict des constructions neuves, etc.) et le changement social des campagnes britanniques ainsi issu d'une forme de sélection basée sur l'aisance financière des ménages (Cloke, 1979; Parsons, 1980; Little, 1987). Par la suite, par-delà la richesse des travaux menés au Royaume-Uni ou aux États-Unis, deux grilles ou entrées analytiques ont semble-t-il été privilégiées. D'une part, la notion d'inégalité étant intimement liée à celle de gentrification, les investigations relatives à la gentrification rurale ont mis en évidence le fait qu'en tant que membres de certaines catégories ou classes sociales, et sans que ce ne soit toujours intentionnel, les gentrifieurs impliqués pouvaient agir en tant que tels parce qu'ils étaient mieux dotés en diverses formes de capital que les populations locales, voire que d'autres ménages susceptibles d'être eux aussi attirés par les mêmes campagnes (Cloke et al., 1998; Phillips, 1993). D'autre part, de nombreuses recherches ont plutôt mis l'accent sur les liens entre « environnement" au sens large, sans forcément le qualifier ainsi, et le processus de gentrification (Smith, 1998; Ghose, 2004; Hines, 2007 ; 2012; Bryson et Wykof, 2010).

Dans le cadre d'un projet ANR ORA+ (iRGENT) consacré à la gentrification rurale aux Etats-Unis, en France et au Royaume-Uni, nous avons pu confronter nos recherches respectives, à l'origine conduites indépendamment les unes des autres ${ }^{1}$. C'est en

1. En France il s'agit des recherches doctorales réalisées par G. Tommasi (2014) sur les recompositions socio-territoriales dans la Montagne limou- partageant nos observations empiriques, donc de manière très inductive, qu'est apparu l'intérêt de réfléchir à une plus grande articulation théorique entre les deux approches de la gentrification rurale évoquées plus haut, c'est-à-dire par l'environnement et par la notion de capital. Nous considérons ici l'environnement dans une acception large, en tant que construction sociale et politique née de l'interaction entre une matérialité biophysique et les représentations dont elle fait objet et dont elle est en même temps projection. L'environnement est donc la combinaison de réalités naturelles, de perceptions et de projections individuelles et collectives sur ces mêmes réalités, ainsi que des influences mutuelles entre réalités et perceptions/projections. La notion de capital est, elle, mobilisée dans une perspective d'inspiration bourdieusienne. Plus encore qu'à travers l’inégale distribution du capital économique, social et culturel et/ou la relation des gentrifieurs à l'environnement, l'idée ici défendue serait que les modalités, causes et conséquences de la gentrification rurale peuvent être analysées et interprétées au regard d'une espèce spécifique de capital, à savoir le capital environnemental.

De fait, l'émergence, voire la multiplication de nouvelles formes de capital, complémentaires des trois espèces originelles (ou quatre selon qu'on y ajoute le capital symbolique), n'est pas sans poser question. Certains, comme Ripoll et Veschambre (2005) vis-à-vis du capital spatial esquissé par Lévy (2003), s'y opposent assez frontalement. D'autres en revanche soulignent les conditions selon lesquelles, dans le respect du cadre théorique bourdieusien, de nouveaux capitaux pourraient émerger, qu'il s'agisse de formes réellement autonomes ou au contraire combinées, voire déclinées depuis les trois principales (Cailly, 2007; Neveu, 2013).

sine. L'enquête, auprès de 71 «nouveaux » et « anciens » habitants, élus et responsables associatifs, a exploré les formes de sociabilités et les pratiques spatiales (notamment à travers l'utilisation de cartes mentales) qui se développent dans un contexte de mobilité et qui révèlent l'émergence de nouvelles inégalités sociales. Au Royaume-Uni, il s'agit de travaux d'abord entrepris dans la région du Lake District à la fin des années 2000 (Richard, 2009) et ensuite élargis à la Grande-Bretagne à l'occasion de la rédaction d'un mémoire d'HDR (Richard, 2017). Ce dernier mobilise d'autres corpus, notamment statistiques mais également de nature qualitative par le biais de relevés paysagers réalisés sur les terrains supports aux publications anglo-britanniques consacrées à la gentrification rurale. Pour le Montana enfin, il s'agit des travaux de thèse engagés par G. Saumon qui interroge l'élaboration de récits partagés sur l'environnement comme composante essentielle des dynamiques socio-territoriales constatées dans l'Ouest du Montana. L'enquête, réalisée sur quatre mois en 2014 et 2015, repose notamment sur 186 entretiens, au cours desquels 77 cartes mentales ont été recueillies. 
À cet égard, les recommandations didactiques de Neveu nous ont guidés pour élaborer et proposer l'hypothèse d'un capital environnemental (Richard et al. 2015; Richard, 2017). De manière très générique, celui-ci pourrait être défini comme l'ensemble des investissements dans l'environnement réalisés par les acteurs sociaux, selon leurs représentations et systèmes de valeurs spécifiques, dans le but de satisfaire leurs desseins et intérêts. Conformément aux précautions énoncées par Neveu, sa validation théorique suppose néanmoins quelques prérequis, y compris empiriques, que sont l'existence d'un champ environnemental structuré autour et à partir de valeurs éponymes, la convertibilité du capital environnemental avec les autres formes de capital, ainsi que la mobilisation ou l'implication du capital environnemental dans des rapports inégalitaires, voire des logiques de domination au sein de la société. En l'occurrence, au-delà de leur formulation théorique et consubstantielle à l'idée d'un capital environnemental, ces diverses notions ont surtout révélé toute leur puissance heuristique lorsque nous les avons assemblées et mises en œuvre en tant que cadre d'analyse « global » des dynamiques de gentrification que nous avions chacun observées sur nos terrains respectifs.

L'objectif de cet article n'est pas d'analyser ou de décrire globalement les dynamiques et modalités de la gentrification rurale dans la Montagne limousine, dans le Montana ou en Angleterre : il vise plutôt à restituer la démarche inductive qui, à partir de nos expériences de terrain, nous a incités à conjuguer les deux grandes approches de la gentrification rurale, pensées jusqu'alors indépendamment (soit par l'environnement, soit par les capitaux). Dans cette perspective, il s'agit plus précisément de considérer le capital environnemental comme une grille de lecture permettant d'appréhender cette double composante de la dynamique de gentrification rurale, et de montrer à partir de nos trois cas d'étude qu'elle est potentiellement applicable à l'ensemble des territoires concernés par ce processus.

Dans un premier temps, nous proposons ainsi d'esquisser un premier pont théorique entre gentrification et capital environnemental, en soulignant d'une part la place consacrée à l'environnement dans la littérature sur la gentrification rurale et dans le processus lui-même, et d'autre part les potentialités d'une approche par le capital dans ce champ de recherche. Dans un deuxième temps, après les avoir présentés, notamment en exposant en quoi leur caractère emblématique a pu y entrainer ou encourager des formes de gentrification rurale, nous mobiliserons nos trois terrains de recherche pour illustrer de manière concrète la manière dont les gentrifieurs mettent leurs valeurs environnementales en actes à travers de nombreuses formes d'investissements, formes souvent similaires en dépit de contextes géographiques dissemblables. Dans un troisième temps, toujours guidés par les préconisations méthodologiques et théoriques de Neveu, nous proposerons d'interpréter les choix et stratégies des gentrifieurs comme autant d'opérations de conversion de divers types de capitaux en direction du capital environnemental. Ainsi, la convertibilité du capital environnemental peut être activée par les individus et ménages pour affirmer leur position au sein de la hiérarchie sociale et conforter leur vision de l'environnement.

\section{COMbiner DEUX APPROCHES CONCEPTUELLES : GENTRIFICATION RURALE ET CAPITAL ENVIRONNEMENTAL}

Objet scientifique relativement bien identifié dans le monde anglo-saxon, en tant que concept et processus géographique, la gentrification rurale n'est encore qu'émergente dans la géographie française. Notre objectif ici n'est de revenir, ni sur la gentrification elle-même, ni sur la littérature qui la concerne (Richard, 2017), mais plutôt de mettre en lumière la place de l'environnement dans cette littérature : en effet, que l'environnement soit considéré en tant que nature, cadre résidentiel, aménités paysagères, objet de pratiques ludiques ou récréatives, voire synonyme de bonne qualité sanitaire, il est toujours au cœur des dynamiques de gentrification. Ce constat nous a ainsi conduits à combiner les approches par l'environnement et par la gentrification, afin d'expliquer les transformations socio-spatiales des espaces ruraux à forte aménité. L'association de ces deux approches nous a permis par ailleurs de théoriser l'émergence d'un capital environnemental : un actif social qui, ajouté aux autres formes de capital, permet aux gentrifieurs, à travers l'imposition de pratiques et valeurs environnementales, d'assoir leur position dominante dans 
les campagnes qu'ils investissent et de s'approprier progressivement ces espaces, de manière matérielle et/ou symbolique. Ainsi, cette partie consiste précisément à interroger la place de l'environnement, d'une part dans les recherches conduites sur la gentrification rurale depuis les années 1970, et d'autre part dans le processus lui-même. Cette rapide mise en perspective épistémologique et empirique nous conduira ensuite à énoncer les termes de l'articulation entre les notions de capital environnemental et de gentrification rurale.

\section{Gentrification rurale, environnement et espaces emblématiques}

En premier lieu, en dépit de ce que pourrait laisser croire la littérature existante, la gentrification rurale n'est pas un avatar de la gentrification urbaine. Si Glass (1964) est considérée aujourd'hui comme l'inspiratrice voire la fondatrice des recherches sur la gentrification urbaine, Pahl (1965) est indiscutablement son alter ego (et contemporain) ruraliste. Cela étant, les deux premiers auteurs à avoir explicitement travaillé sur la gentrification rurale l'ont fait à propos des campagnes anglaises des années d'aprèsguerre (Parsons, 1979; 1980; Cloke, 1979; 1983). Questionnant tous les deux les liens entre changement social des campagnes anglaises et politiques d'aménagement et de planification (par le biais de la key settlement policy), ils constatent le même processus de substitution des populations modestes, souvent amenées à déménager vers les villes plus ou moins proches, par des ménages issus des middle classes, soit actifs et commuters, soit retraités. Ce lien entre planification et gentrification a d'ailleurs longtemps influencé la nature de l'attention portée au processus (Little, 1987; Marsden et al., 1993).

Les recherches ont cependant pris une autre orientation, plus empreinte de sociologie, à la fin des années 1980 par le truchement notamment d'un programme de recherche collectif piloté par P. Cloke et consacré aux modalités d'implantation de différentes fractions des middle classes dans les campagnes anglaises et galloises. C'est d'ailleurs à l'issue de ce programme que M. Phillips (1993) s'est appuyé sur les débats théoriques qui opposaient alors les partisans des explications de la gentrification soit par la production, soit par la consommation ${ }^{2}$, pour initier les premières convergences théoriques entre gentrifications rurale et urbaine, jusque-là étanches l'une à l'autre. À l'occasion de cet article, M. Phillips a ainsi pu donner un certain crédit à la lecture de la gentrification en tant qu'expression d'une « circulation inégale du capital » (Phillips, 1993, p. 125) autant qu'il a mis en évidence la pluralité des processus de gentrification au regard des types d'individus et de ménages impliqués.

Du reste, cette approche par la production, et en particulier via l'hypothèse du rent gap ${ }^{3}$, a également été privilégiée par E. Darling (2005) pour interpréter les dynamiques de gentrification (massivement portées par le développement des résidences secondaires) dans le Parc des Adirondacks aux États-Unis. Cependant, en portant une attention toute particulière au rôle de ce qu'elle qualifie de "Wilderness ", c'est-à-dire aux aménités naturelles, et singulièrement celles qui sont liées à la présence des innombrables lacs au sein du parc, E. Darling souligne explicitement l'intérêt d'intégrer «la nature » au cadre d'analyse du processus de gentrification rurale aux États-Unis.

Ce faisant, elle s'inscrit dans les pas de Cloke et al. (1995) qui avaient déjà identifié l'accès à la nature et/ou à la ruralité (et aux sociabilités spécifiques que le terme impliquerait) comme un moteur puissant de la colonisation des campagnes britanniques par les middle classes. Plus encore, en proposant le terme de greentrification pour qualifier les dynamiques de la petite ville d'Hebden Bridge et de ses environs, Smith (1998) a fait des relations entre gentrification et environnement une problématique fondamentale (Smith et Phillips, 2001 ; Phillips, 2002 ; 2005). Le néologisme tend à souligner «the demand for, and perception of "green" residential space from inmigrants households » (Smith, Phillips, 2001, p. 457) et ainsi à replacer les observations empiriques dans le débat théorique quant aux origines de la gentrification. En l'occurrence, cette vision d'une

\footnotetext{
2. Dans cette controverse, alors qu'une partie des chercheurs explique la gentrification en mettant l'accent sur la demande (la « consommation »), c'est-à-dire les choix culturels et les logiques résidentielles des nouvelles élites urbaines (Ley, 1980), d'autres mettent l'accent sur l'offre ( « la production ») en soulignant le rôle des marchés fonciers et immobiliers comme producteurs de gentrification (N. Smith, 1987). Les deux positionnements ne sont toutefois pas inconciliables, les deux approches pouvant au contraire être considérées complémentaires (Hamnett, 1997).

3. Pour une synthèse en français de ces débats, cf. par exemple C. Hamnett, 1997.
} 
gentrification rurale due à des goûts, à des modes et objets de consommation en lien avec l'environnement (tels que des paysages, la nature, la quiétude rurale, un milieu sain et sûr, etc.) et propres à des catégories socio-culturelles spécifiques est désormais partagée par la plupart des chercheurs anglo-saxons. À cet égard, les travaux américains consacrés aux parcours migratoires et plus généralement aux divers types de ménages contribuant à la gentrification de l'Ouest américain, notamment du Montana, ont permis de réaffirmer la composante environnementale du processus, en y intégrant par exemple les activités récréatives de plein air (Hines, 2007), ce qui a amené certains auteurs à proposer les notions "d'expérience » (sensible/environnementale) et de "post-tourisme » (Hines, 2010) pour expliquer la bascule de l'Old West vers un New West post-productif marqué du sceau des gentrifieurs (ibid.; Bryson et Wickoff, 2010). Quant aux recherches françaises ayant convoqué la notion de gentrification rurale, plus ou moins consciemment inscrites dans le paradigme de la renaissance rurale ou de la néo-ruralité, qu'elles aient ou non bénéficié de l'antériorité des références précitées, elles ont, pour la plupart ${ }^{4}$, elles aussi pointé la composante environnementale de l'attractivité renouvelée des campagnes françaises. Autrement exprimé, que ce soit de manière implicite pour ceux qui ont choisi une entrée migratoire (Raymond, 2005; Cognard, 2006; Pistre, 2012; Desponds, 2007) ou explicite (Richard et al., 2014b), c'est bien l'hypothèse de la greentrification et, par-delà, une approche de la gentrification par la demande qui ont été privilégiées.

En l'occurrence, si la littérature sur la gentrification rurale accorde une place fondamentale à l'entrée environnementale, il faut préciser sa place dans le processus lui-même. En considérant le fait que le processus de gentrification est en première instance fondé sur des mouvements de population (Smith, 2002), Richard et al. (2014b) ont ainsi proposé d'appréhender les modalités d'articulation entre « l'environnement » et chacune des principales étapes des trajectoires résidentielles et migratoires (figure 1 - planche XI). En amont de la migration (figure la - planche XI), l'environnement intervient en tant qu'image socialement construite, à

4. À l'exception notable de Perrenoud (2008) qui, sociologue, s’intéresse aux positionnements et stratégies des artisans du bâtiment, en tant «qu'agents » de la gentrification rurale. travers les représentations véhiculées par les productions culturelles et les mass media (journaux, émissions télévisés, romans...). L'exemple de Postman Pat est à ce titre intéressant : pour Horton (2008), les personnages, les paysages, la société présentés dans ce dessin animé ont contribué à modeler l'imaginaire de la campagne anglaise. De même, d'autres productions culturelles (magazines, romans, manifestations locales, etc.) produisent des représentations de la ruralité, sur laquelle les ménages projettent leurs aspirations résidentielles. Ces aspirations se concrétisent lors de la deuxième étape : ce moment correspond à l'itinéraire migratoire et résidentiel des individus, et c'est en tant que matérialité biophysique (paysages appréhendés à diverses échelles) que l'environnement concourt, parmi d'autres facteurs et critères, à orienter les préférences des ménages pour telle ou telle localisation, tel ou tel type et esthétique d'habitat (figure $1 \mathrm{~b}$ - planche XI). L'hétérogénéité des profils des gentrifieurs se reflète ici dans le choix de différents contextes résidentiels, avec des critères, liés au logement, au paysage, au contexte social, qui influencent leur parcours. Enfin, l'environnement intervient à l'issue de l'implantation des individus et groupes sociaux, c'est-à-dire dans les « temporalités avales » du processus de gentrification (Richard et al., 2014b). Les nouveaux habitants influent plus ou moins sensiblement sur l'environnement, d'une part par leur impact direct sur les structures et compositions paysagères (en priorité à l'échelle domestique, à travers l'esthétisation et la mise en scène des jardins par exemple) (figure 1c - planche XI). D'autre part, l'impact est parfois plus indirect et se manifeste sur les politiques territoriales et la gestion de l'environnement (influence sur les règles d'urbanisme ou les périmètres de protection ${ }^{5}$ ). Ces actions participent à conforter les images et représentations qu'ils en avaient lors de la première étape, et alimentent ainsi le cycle des relations environnementgentrification.

\footnotetext{
5. Dans de nombreuses communes par exemple, l'implication des nouveaux habitants au sein des conseils municipaux ou dans les parish councils britanniques leur permet d'influencer l'élaboration des plans ou des règles d'urbanisme, bien souvent en faveur d'une limitation des nouvelles constructions et de formes de protections environnementales. Dans une certaine mesure, ils instrumentalisent ainsi les documents de planification (Little, 1987; Charmes, 2011) pour figer le paysage tel qu'il était au moment de leur investissement résidentiel, et satisfaire leur vision idyllique d'une campagne isolée et intime (Kondo et al., 2012).
} 
Mais cette capacité à peser sur la composante environnementale d'un territoire n'est pas également donnée à tous les habitants. Très directement liée aux divers capitaux dont ils sont dotés, elle s'ajoute aux autres indicateurs de différenciation au sein des populations rurales et interroge donc de front la question des inégalités sociales. Cette perspective nous invite alors à préciser en quoi le capital environnemental se pose en actif social déterminant dans le processus de gentrification rurale.

\section{L'hypothèse du capital environnemental dans le processus de gentrification rurale}

Fortement guidée par l'observation empirique des mutations des campagnes anglaises à l'œuvre dans le courant des années 1950-1970, l'étude de la gentrification rurale s'est enrichie très tôt des acquis de la sociologie bourdieusienne. En premier lieu, considérant le lien ontologique qui unit gentrification et inégalités sociales, la théorie consistant à concevoir diverses espèces de capital pour mieux déconstruire les processus à l'origine des inégalités au sein de nos sociétés s'est très mécaniquement imposée aux ruralistes d'Outre-Manche explorant la question des classes sociales dans les campagnes (Cloke et Thrift, 1987). Elle a été d'autant mieux reçue qu'elle a permis d'appréhender l'hétérogénéité des populations concernées par les migrations villecampagne. Concrètement, selon la composition de leurs portefeuilles d'actifs, les gentrifieurs accèdent aux espaces ruraux via diverses " channels of entry » (Phillips, 1993; 2002; Cloke et al., 1998). Sur la même base conceptuelle, K. Halfacree (2011) distingue lui aussi les gentrifieurs « conventionnels» ou «mainstream » de ceux qui sont plutôt acteurs d'une « radical rural gentrification » associée au mouvement Back to the Land. En second lieu, la vision bourdieusienne du capital et des classes sociales a séduit les ruralistes britanniques en ce qu'ils ont trouvé dans la notion d'habitus une clé de lecture intéressante pour appréhender d'un seul regard à la fois la recherche et la mise en œuvre d'un style de vie propre à la middle class, voire aux différentes fractions qui la composent, et les paysages ruraux dans lesquels s'incarnent ces styles de vie.

Par ailleurs, les apports conceptuels de P. Bourdieu ont paradoxalement permis de réunir ou de faire aisément cohabiter les deux approches de la gentrification, par l'offre et la demande. En effet, la première a pu être satisfaite par le fait que le capital économique reste un actif majeur dont on peut lire les flux inégaux à destination des espaces ruraux, et ce, y compris en identifiant des processus et agents similaires à ceux décrits dans les théories classiques du rent gap. Quant à la seconde, elle s'est également trouvée confortée par l'habitus et le style de vie mentionnés plus haut (Smith, 1998).

Quoi qu'il en soit, pour en venir au lien entre la gentrification rurale et le capital environnemental, on peut donc partir du principe selon lequel des individus et ménages suffisamment pourvus en capitaux économiques, sociaux, et culturels s'installent dans les campagnes attractives. Par ailleurs, en théorie, plus elles sont attractives plus elles nécessitent des groupes sociaux qu'ils soient bien dotés, et sont donc sélectives. Une fois installés, du fait de leur position dominante (avec toutes les nuances possibles et nécessaires) au sein des sociétés locales, les gentrifieurs sont en mesure d'imposer tout ou partie de leur conception de la campagne, y compris à ceux qui ne la partagent pas ou que partiellement. Or, dans la mesure où l'environnement est précisément au cœur de ces conceptions de la campagne, qu'il apparaît comme un facteur central, peut-être la pierre angulaire, du processus de gentrification rurale, s'est posée pour nous l'hypothèse d'un capital environnemental qui viendrait compléter les autres espèces de capital (économique, culturelle, sociale, voire symbolique). Alors que cet argumentaire a été développé ailleurs d'un point de vue théorique et général (Richard et al. 2015; Richard, 2017), la suite de la présente contribution va plutôt consister à montrer en quoi elle offre une grille de lecture pertinente de la gentrification rurale.

\section{Valeurs environnementales et pluralité des processus de gentrification rurale}

Sans pouvoir le détailler complètement ici, il est nécessaire de rappeler quelques éléments clés de l'approche bourdieusienne des capitaux et de les décliner pour le cas spécifique du capital environnemental. En premier lieu, l'existence d'une quelconque forme de capital présuppose celle d'un champ issu de valeurs socialement reconnues et légitimées qui lui sont associées (Bourdieu, 2000; Cailly, 2007; Neveu, 2013). Au capital environne- 
mental est donc associé un champ constitué de multiples valeurs liées à l'environnement et vis-àvis desquels les individus et groupes se positionnent, que ce soit en les négligeant, en les contestant, en y adhérant de manière plus ou moins vague, ou encore en les promouvant y compris à travers des actes. En l'occurrence, le principe d'un champ environnemental nous semble d'autant plus justifié que bien au-delà de la question de la gentrification rurale, la montée en puissance et la reconnaissance des enjeux sociaux, (géo)politiques, économiques, symboliques, etc., liés à l'environnement sont chaque jour un peu plus avérées (Keucheyan, 2014 ; Richard et al., 2015). Ainsi, c'est en fonction de leurs objectifs, de leurs intérêts, de leurs possibilités respectives que les acteurs réalisent des investissements sur la base de valeurs relatives par exemple au cadre de vie, à l'habitabilité des lieux (Blanc, 2008), aux paysages, aux enjeux écologiques, etc., valeurs qui s'incarnent concrètement dans l'espace. Cependant, avant de présenter plus avant cette lecture de la gentrification rurale, il est nécessaire de familiariser le lecteur avec chacun des trois terrains illustrant notre propos. Cette section sera l'occasion de montrer la pluralité des formes possibles de la gentrification rurale, ne serait-ce qu'au travers des ménages impliqués, des degrés d'intensité ou de l'échelle d'observation du processus.

\section{Trois campagnes emblématiques inégalement gentrifiées}

Les campagnes anglaises, le Montana et la Montagne limousine incarnent à travers leurs aménités, leurs ambiances paysagères, les représentations et les pratiques qui leur sont associées, un emblème de la nature et de l'environnement (ou des rapports à celui-ci). De par les flux migratoires qui les nourrissent, devenus significatifs selon des temporalités différentes d'un terrain à l'autre, elles ont été ou sont aujourd'hui investies par de nouvelles populations qui contribuent à en modifier la structure socio-économique, les paysages, et au final, l'image.

En investiguant, chacun de son côté, ces trois terrains de recherche, et échangeant sur nos résultats, nous nous sommes rendu compte que, malgré l'hétérogénéité des formes et de l'intensité de la gentrification, nous partagions des clés d'analyse nous permettant de lire les transformations de ces espaces ruraux. L'hétérogénéité des groupes sociaux concernés, les spécificités environnementales et paysagères, les échelles forcément différentes, les méthodes adoptées pour nos recherches, nous ont conduits non pas à une approche comparative, impossible rétrospectivement mais, de manière inductive, à identifier une grille de lecture commune des processus que nous observions.

Parce qu'elles ont été les premières touchées, la présentation des terrains peut démarrer par les campagnes britanniques, notamment anglaises, dont la construction remonte au double processus d'urbanisation et d'industrialisation, amorcé dès le $\mathrm{XVIII}^{\mathrm{e}}$ siècle mais violemment conforté au XIX ${ }^{\mathrm{e}}$ siècle. À mesure que la pollution de l'eau et de l'air s'aggravait dans les villes, par ailleurs en pleine expansion démographique et spatiale, les espaces qui en restaient préservés ont à l'inverse bénéficié d'une image de plus en plus positive. Parallèlement, pardelà cette dimension sanitaire et plus largement environnementale, le mouvement romantique (dont W. Wordsworth avec le Lake District est souvent présenté comme la figure de proue) a contribué à faire des campagnes non seulement « un paysage », mais également l'ultime incarnation de la «nature » (Bunce, 1994; Mathis, 2010; Berque, 2010). Dès lors, bénéficiant d'une conjonction de facteurs favorables, les espaces ruraux et/ou naturels sont devenus très attractifs aux yeux des catégories socioculturelles à la fois sensibles aux invites sentimentalistes et en mesure de s'offrir une résidence permanente, saisonnière ou ponctuelle « hors la ville ». Si le mouvement ne s'est pas démenti dans la première moitié du $\mathrm{xx}^{\mathrm{e}}$ siècle, il s'est encore accéléré dans les décennies suivantes qui ont vu les middle classes, puis les services classes investir très massivement ces territoires (Newby, 1979; Hoggart, 1997). Désormais très attractives, sous pression foncière et démographique mais en même temps protégées (autant par les règles locales d'urbanisme que par des périmètres de protection divers tels que les Area of Natural Outstanding Beauty [AONB] ou les Parcs Nationaux), les campagnes sont devenues des espaces de «nature » nécessitant pour ceux qui aspirent à y vivre, y compris à temps partiel pour les weekenders, de disposer de moyens financiers très conséquents. Désormais, dès lors qu'elles sont 
aisément accessibles depuis les centralités urbaines ${ }^{6}$ (où d'ailleurs une partie des gentrifieurs se rend régulièrement pour le travail), et/ou qu'elles offrent des aménités environnementales qui sortent un tant soit peu de l'ordinaire, les campagnes anglaises sont socialement très sélectives. La figure 2 (planche XII) montre ainsi l'ampleur de la « colonisation » des campagnes (Phillips, 1993 ; 2007) et plus généralement des espaces « naturels » britanniques par les catégories socioprofessionnelles généralement décrites comme constitutives des middle classes (Hoggart, 1997; Phillips, 2007). À l'issue d'un processus observé dès les années 1960, cellesci sont proportionnellement surreprésentées dans la majorité des espaces ruraux et y constituent même plus d'un actif sur deux dans de vastes portions. Plus encore, traduisant une dynamique longue de plus d'un demi-siècle, le tableau 1 précise que plus la morphologie de l'habitat permet de proximité aux aménités environnementales et paysagères (habitat isolé, hameaux, voire villages plutôt que bourgs), plus ils sont investis par les plus qualifiés ${ }^{7}$. Si les middle classes ont progressivement modelé les campagnes à l'image de ce qu'elles souhaitaient y trouver, les campagnes sont désormais perçues au sein de la société britannique comme co-constitutives des middle classes et de leur identité socio-culturelle (Cloke et al., 1998).

Dans le Montana, le processus de gentrification, relativement récent, ne peut se saisir que dans l'épaisseur historique des représentations de l'environnement qui le nourrissent. Espace emblématique de la Wilderness et de la Frontier, le Montana incarne en effet un Ouest de la nature sauvage, terreau de l'identité américaine. Si la représentation collective de l'Ouest est inséparable de la dynamique de front pionnier, les mobilités qu'il suscite depuis les années 1970, et surtout 1990, représentent une nouvelle manifestation de cet esprit de conquête. Ce dernier repose sur un rapport intense et ambigu à la nature sauvage, entre fascination (contemplation, source

6. Soulignons qu'outre-Manche, que ce soit du point de vue des nomenclatures spatiales officielles (du type Rural Urban Classification 2011) ou des représentations sociales et culturelles, les espaces qui correspondraient au périurbain en France relèvent du rural.

7. Les NS-SEC 1, 2 et 4 correspondent respectivement, d'abord aux chefs d'entreprises de plus de 25 salariés, cadres, professions libérales et intellectuelles supérieures, puis aux cadres moyens et professions intellectuelles, et enfin aux chefs d'entreprise de moins de 25 salariés et autres travailleurs indépendants. Très hétérogène, cette dernière catégorie est associée à la " petite bourgeoisie » par les géographes et sociologues britanniques. d'inspiration artistique, retraite...) et logique de domination (mise en parc, activités extractives...). L'environnement occupe ainsi aujourd'hui une place déterminante dans les dynamiques migratoires du Montana, et il faut souligner l'attraction qu'exercent d'une part la pêche à la mouche ${ }^{8}$ et d'autre part les montagnes Rocheuses. Entre la pratique d'activités de pleine nature (randonnée, ski, kayak...), l'accès aux espaces naturels protégés (Parcs Nationaux de Yellowstone et de Glacier, National Forests, Wilderness Areas...) et un cadre paysager quotidien idyllique, la gentrification du Montana dépend de la nouvelle attention portée aux aménités environnementales. Ce processus vient nourrir une profonde mutation territoriale, dont le principal facteur repose sur la manière dont l'environnement est réinvesti aujourd'hui (Saumon, 2016), passant de ressource naturelle à extraire, dans une perspective productiviste, à paysage à valoriser, dans une perspective post-productiviste : cette réinterprétation est au cœur de la transformation de l'Old West - l'Ouest de l'économie extractive - en New West - l'Ouest de l'économie d'aménités. Pour autant, ce processus de gentrification n'est pas uniforme dans le Montana, à l'échelle régionale d'abord - entre une partie est encore dévolue aux activités primaires et secondaires, et une partie Ouest dont l'essor est de plus en plus fondé sur le développement touristique et résidentiel; à l'échelle locale ensuite - entre des sites particulièrement attractifs d'une part, et des sites pollués et répulsifs pour les gentrifieurs d'autre part (figure 3 - planche XIII). Le New West est alors à interroger comme territoire fragmenté : loin de pouvoir qualifier des dynamiques qui seraient égales et homogènes sur l'ensemble du territoire, il prend la forme, pour J. Dwight Hines, d'un archipel (Hines, 2012). Le processus de gentrification ne concerne ainsi que quelques territoires élus, chacun drainant des profils de gentrifieurs spécifiques, établis dans des quartiers en renouvellement ou dans des immenses propriétés sur les hauteurs des villes. À Missoula ou Bozeman par exemple, les gentrifieurs, disposant d'un fort capital culturel, sont à la recherche de modalités de consommation alternatives de l'environnement qu'ils peuvent satisfaire dans des commerces et des cafés branchés. Dans les stations de villégiatures aux portes des Parcs

8. Particulièrement depuis l'adaptation par Robert Redford du récit de Norman MacLean A River Runs Through It (MacLean, 1976; Redford, 1992). 


\begin{tabular}{|l|r|c|c|c|c|}
\hline $\begin{array}{l}\text { Rural Urban Classification } 2011 \\
\text { (RUC1 1) }\end{array}$ & $\begin{array}{c}\text { Tous actifs } \\
16-74 \text { ans }\end{array}$ & $\begin{array}{c}\text { NS SEC 1 } \\
\text { (en \%) }\end{array}$ & $\begin{array}{c}\text { NS SEC 2 } \\
\text { (en \%) }\end{array}$ & $\begin{array}{c}\text { NS SEC 4 } \\
\text { (en \%) }\end{array}$ & $\begin{array}{c}\text { NS SEC 1, } \\
\text { 2 et 4 (en \%) }\end{array}$ \\
\hline Rural town and fringe & 3497964,00 & 11,7 & 24,2 & 11,8 & 47,6 \\
\hline Rural town and fringe in a sparse setting & 210999,00 & 7,3 & 20,6 & 15,5 & 43,3 \\
\hline Rural village and dispersed & 2848370,00 & 14,6 & 26,3 & 16,7 & 57,5 \\
\hline $\begin{array}{l}\text { Rural village and dispersed in a sparse } \\
\text { setting }\end{array}$ & 375806,00 & 9,4 & 22,5 & 23,4 & 55,3 \\
\hline All Rural & $\mathbf{6 9 3 3 \mathbf { 1 3 9 , 0 0 }}$ & $\mathbf{1 2 , 6}$ & $\mathbf{2 4 , 8}$ & $\mathbf{1 4 , 5}$ & $\mathbf{5 2 , 0}$ \\
\hline Urban city and town & 16711282,00 & 10,5 & 22,3 & 9,4 & 42,2 \\
\hline Urban city and town in a sparse setting & 95319,00 & 6,4 & 19,0 & 11,9 & 37,3 \\
\hline Urban major conurbation & 12429865,00 & 11,9 & 23,0 & 9,5 & 44,4 \\
\hline Urban minor conurbation & 1249072,00 & 9,0 & 19,6 & 8,5 & 37,1 \\
\hline All Urban & $\mathbf{3 0 4 8 5 5 3 8 , 0 0}$ & $\mathbf{1 1 , 0}$ & $\mathbf{2 2 , 5}$ & $\mathbf{9 , 4}$ & $\mathbf{4 2 , 8}$ \\
\hline Angleterre et Pays de Galles & $\mathbf{3 7 4 1 8 6 7 7}$ & $\mathbf{1 1 , 3}$ & $\mathbf{2 2 , 9}$ & $\mathbf{1 0 , 3}$ & $\mathbf{4 4 , 5}$ \\
\hline
\end{tabular}

Tableau 1 : Distribution des NS-SEC supérieures (middle classes) dans les espaces ruraux et urbains (Angleterre, Pays de Galles, 2011). Sources : ONS Census 2011 , calculs auteurs.

Distribution of NS-SEC 1, 2, 4 (middle classes) in rural and urban spaces (England and Wales, 2011). Sources: ONS Census 2011, authors' calculations.

Nationaux, une élite économique vient pratiquer des sports de pleine nature en appréciant l'entre-soi que ces microcosmes favorisent.

En Montagne limousine, le processus de gentrification est également récent et inégal dans l'espace, puisqu'associé aux migrations résidentielles à destination de ce territoire de moyenne montagne. Amorcées avec les mouvements néo-ruraux des années 1970, les migrations se sont intensifiées dans les deux dernières décennies par l'intermédiaire de migrants nationaux (originaires de la Région parisienne ou des grandes villes des régions limitrophes) et Nord-Européens (notamment Britanniques). Il s'agit de ménages en âge d'être actifs, même si la présence de retraités est significative, qui disposent de capital culturel (ils sont plus diplômés que la moyenne du territoire) et social (fonctionnement en réseau, nombreux liens en dehors du territoire), le capital économique n'étant pas toujours le plus distinctif. Cela a contribué à l'affirmation du territoire comme emblématique d'une ruralité alternative et en renouvellement (Tommasi, 2014) ${ }^{9}$.

Synonyme d'une nature préservée, riche en biodiversité (par ailleurs valorisée dans le cadre du PNR de Millevaches et d'autres périmètres de protec-

9. Richard et al. (2014b) ont à ce propos proposé le terme d' " altergentrifieurs ", proche du profil des marginal gentrifiers identifié en Angleterre (Phillips, 1993). tion, comme le montre la figure 4 - planche XIV), l'environnement naturel occupe une place importante dans la dynamique migratoire : il joue dans la décision de migrer et dans le choix d'installation, et est ensuite vécu en tant que nature à pratiquer (activités récréatives et culturelles, sports de nature) ou en tant que ressource (exploitations agricoles et forestières). Par ailleurs, de nombreux habitants s'investissent, au niveau associatif ou politique, dans la gestion et la protection de l'environnement. Cet investissement multiforme se manifeste tant dans les choix individuels (consommation en circuit court de proximité, matériaux de construction écologiques...) que dans des démarches collectives (associations d'éducation environnementale, arrêtés municipaux réglementant les pratiques de gestion des ressources, écoquartiers...).

Le processus de gentrification se manifeste avec une intensité relative et très inégale dans l'espace, et la composition sociale du territoire reste par ailleurs diversifiée : des ménages modestes sont encore présents, notamment grâce à un bâti abordable. Cependant, les impacts territoriaux du processus sont tangibles, d'abord à l'échelle locale : les gentrifieurs sont à l'origine d'initiatives empreintes d'une esthétique nouvelle et valorisant des aspects sociaux et environnementaux qui ont impulsé une dynamique économique et sociale : des entreprises qui 
travaillent et valorisent les ressources locales (circuits courts par exemple), des associations qui animent une vie sociale (crèches, accueil de nouvelles populations, services à la personne...) et culturelle (festivals, media locaux...). Mais c'est surtout à une échelle micro-locale que le processus apparait de manière plus évidente (Richard et al., 2014b) : les effets de la gentrification sont alors visibles à l'échelle de hameaux ou de quelques communes particulièrement attractives, où les maisons et leurs environnements proches sont (re)façonnés, notamment à force de rénovations.

\section{Valeurs environnementales et investissements dans le capital environnemental par les gentrifieurs}

Si l'environnement est une dimension fondamentale du processus de gentrification rurale, les formes de sa mobilisation sont souvent variées, correspondant aux différents signifiés que recouvre le terme « environnement » mais également aux valeurs, parfois contrastées, qui lui sont associées. Pour revenir au capital environnemental et aux notions qu'il sous-tend, le champ de l'environnement est constitué de valeurs environnementales, que nous avons regroupées en "sous-champs » thématiques, aux frontières poreuses et en partie superposables. Dans une liste non exhaustive des sous-champs, figurent l'habitat ou l'habitabilité, le paysage et la naturalité, la qualité environnementale et les pratiques écologisantes, les enjeux sanitaires, les composantes productives et postproductives de l'environnement (Richard et al. 2015; Richard, 2017). Dans nos trois terrains, nous avons observé la manière dont les gentrifieurs se reconnaissent dans les valeurs de ces souschamps, et les mobilisent selon des intérêts qui leur sont propres. Cette section vise à montrer en quoi les choix et stratégies des gentrifieurs peuvent être lus et interprétés comme autant d'investissements matériels et symboliques. Pour rester dans le lexique spécifique à la théorie du capital, lorsque les gentrifieurs procèdent à ces investissements, ils se positionnent au sein du champ environnemental et acquièrent du capital du même nom. Quelques exemples peuvent utilement illustrer le propos.

Très concrètement, quel que soit le terrain, les entretiens ont indiqué que les valeurs relatives à l'habitat (le cadre résidentiel, le logement, le bienêtre associé à cet espace) font l'objet d'un investissement important de la part des gentrifieurs. Qu'ils aient investi des villages anglais patrimonialisés et esthétisés, ou des logements plus isolés du Montana ou de la Montagne limousine, les gentrifieurs sont attentifs à leur confort intérieur, à leur « authenticité » architecturale (ranch, cottage, longère traditionnelle) autant qu'à leur environnement domestique et paysager, à leur inscription dans « la nature », ou encore à l'accès à des perspectives paysagères et autres aménités locales (forêts, landes, étangs, etc.). Sur les trois terrains, que ce soit par le biais de rénovations, de travaux, la création de nouvelles ouvertures, les matériaux utilisés locaux et/ ou écologiques, ou par la restructuration des jardins et le remodelage des paysages (figures 1c, figure 5), la plupart des gentrifieurs expriment une relation singulière à l'environnement, parfois une forme d'éthique, témoignant ainsi de valeurs massivement partagées et auxquelles ils montrent par leurs actes qu'ils adhèrent.

D'ailleurs, au-delà de l'habitat, la qualité environnementale et les pratiques écologisantes font quotidiennement l'objet de positionnements affirmés, parfois d'un engagement fort vis-à-vis de la protection de l'environnement. Les gentrifieurs rencontrés dans nos terrains d'études, et plus particulièrement dans le Montana et la Montagne limousine, appliquent à leurs choix et engagements des principes d'éco-responsabilité, de développement durable, de respect des ressources naturelles. Cela se traduit par le soutien ou l'engagement dans des associations ou ONG environnementales : les nouveaux habitants sont nombreux à s'investir dans des associations de protection ou d'éducation à l'environnement, et se positionnent vis-à-vis de politiques de protection ou labellisation. Dans le quotidien, ces valeurs peuvent également se matérialiser par des pratiques visant à réduire l'utilisation de la voiture individuelle en faveur de formes de mobilité alternatives, comme le vélo ou le covoiturage, ou dans des pratiques de consommation privilégiant les circuits de proximité et/ou la consommation de produits biologiques (figure 5b) et ce, pour des motivations de nature explicitement environnementale. Bien que ces valeurs soient également plébiscitées, voire quelques fois revendiquées dans les campagnes anglaises, elles sont souvent intégrées à des 
Figure 5: Exemples d'investissements et valeurs associés au champ environnemental

a. Des chalets de haut standing au cour de la forêt, Whitefish, Montana; b. La valorisation des circuits de proximités et des productions agricoles biologiques, Missoula Farmer's Market, Montana; c. Des espaces extérieurs domestiques fortement investis pour leur contribution esthétique et le sentiment d'unité avec la «nature ", Montagne limousine). Clichés auteurs, 2013, 2014. Example of investments and values associated with the environment

(a) luxury chalets in the forest, Whitefish, Montana; (b) promotion of local food-supply chains and organic farm produce, Missoula Farmers Market, Montana; (c) outdoor domestic spaces highly valued for their aesthetics and the feeling they offer of being at one with nature, Montagne Limousine. Authors' photos, 2013 and 2014.

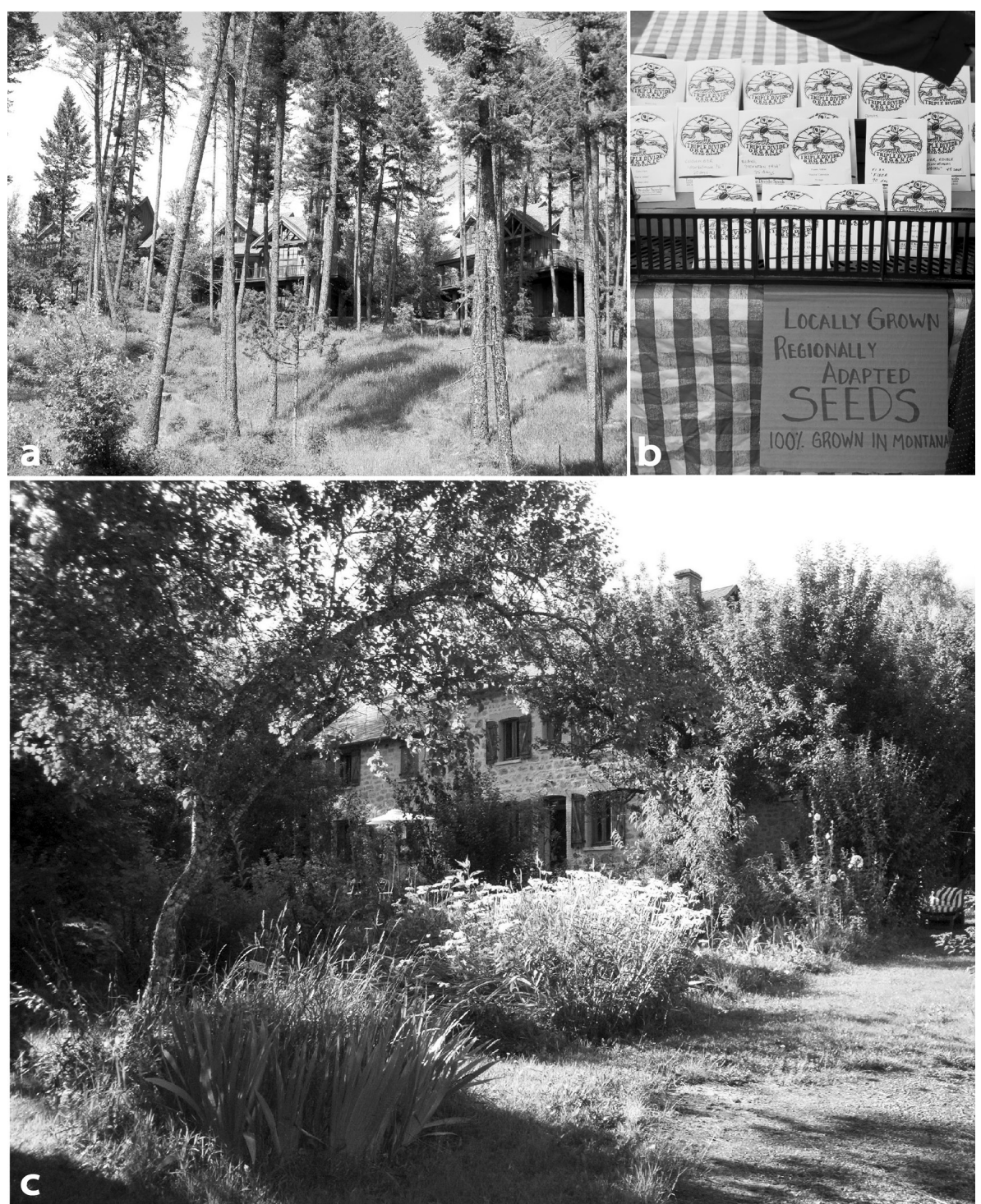

idéaux de protection des paysages, de la nature qui justifient l'opposition à tout ce qui serait susceptible d'y porter atteinte.

La référence précédente aux produits locaux permet par ailleurs d'introduire le sous-champ structuré autour de la composante productive de l'environnement, vue comme une ressource économique, que ce soit dans le cadre d'activités agricoles ou d'exploitations d'autres ressources (forêts, énergies renouvelables...). Concernant les circuits courts, leurs liens avec la gentrification ont déjà été mis en lumière dans le contexte limousin (Richard et al., 2014a). Outre-Manche, Sutherland (2012) décrit l'investissement dans l'activité agricole par les « hobby farmers » et "new country gentlemen », néo-arrivants propriétaires de terres agricoles de petites surfaces, qui produisent parfois uniquement pour un usage personnel et dans un but récréatif. Considérant la ferme comme un espace de jeu, ils s'attachent surtout à produire des aménités environnementales directement consommables, et par là, façonnent le paysage correspondant à un idéal (fermier) de middle class qui leur permet une insertion rapide dans un cercle restreint de pairs. En l'occurrence, si elle mentionne plutôt des ranchers que des gentlemen farmers, la littérature américaine décrit également cette tendance (Friedberger, 1996; Gosnell et Travis, 2005 ; Travis, 2007; Woods, 2016).

Cependant, comme dans de nombreuses campagnes des pays occidentaux, la composante productive de nos terrains de recherche est désormais concurrencée, sinon dépassée, par la dimension 
post-productive de l'environnement. Ce sous-champ regroupe pour nous les valeurs qui font de l'environnement un objet de consommation, un lieu d'agrément, de pratiques touristiques et de loisir. Il est logiquement très investi par les gentrifieurs en quête d'un terrain de jeu pour des pratiques sportives, récréatives, culturelles. En Limousin par exemple, la campagne devient le cadre de manifestations culturelles (théâtre, performances, expositions...) réalisées sur et dans l'environnement. Dans le Montana, de nombreux gentrifieurs s'installent pour profiter des pratiques sportives en plein air (ski, VTT, golf) socialement distinctives, que l'on retrouve au Royaume-Uni (notamment dans les campagnes des parcs nationaux) où elles restent néanmoins concurrencées par la passion du jardinage, autant pour soi que pour les autres (au-delà de la co-visibilité, de nombreux jardins privés sont ouverts à la visite).

Déclinés en quelques-uns des sous-champs constitutifs du champ environnemental, ces exemples de valeurs environnementales investies au sein de nos terrains illustrent non seulement la variété des représentations et usages de l'environnement, mais permettent en outre de souligner la pluralité des gentrifieurs : d'un terrain à l'autre, mais aussi au sein d'un même terrain, le profil des gentrifieurs n'est bien entendu pas homogène. Leurs origines sociales, leurs motivations, leurs attentes vis-à-vis de l'environnement contribuent à différencier leurs pratiques et leurs investissements dans les valeurs environnementales. Cette hétérogénéité se reflète par ailleurs dans le vocabulaire utilisé pour les définir : «mainstream », " altergentrifieurs ", " marginal », « hobby farmers ", ces appellations suggèrent des différences entre gentrifieurs, qui sont toutefois unis par le partage d'un positionnement fort vis-à-vis de l'environnement. Concrétisant leur adhésion à ces valeurs par des actes et des pratiques, ils apparaissent ainsi mieux pourvus en capital environnemental. S'ensuivent alors des rapports de force entre des acteurs dont l'inégale capacité à investir dans l'environnement traduit ou renforce les hiérarchies en place.

\section{Conversion, accumulation DES CAPITAUX ET LOGIQUES DE DOMINATION}

L'approche par les capitaux permet d'éclairer la manière dont les acteurs les mieux dotés main- tiennent leurs positions dans divers champs (économique, social, culturel), au sein desquels se jouent les rapports sociaux et qui, par-delà, structurent la société. Dans cette perspective, le capital environnemental pourrait constituer une espèce supplémentaire de capital (ou à défaut, une « combinatoire des espèces originelles », cf. Neveu, 2013; Richard et al., 2015) susceptible d'expliquer le maintien ou l'aggravation des inégalités socio-environnementales. À cet égard, Neveu souligne la nécessité des opérations de conversion d'une forme de capital en une ou plusieurs autres formes, conversion qui se doit par ailleurs d'être réversible. Là encore, à la lumière des faits observés sur les terrains, il nous est effectivement apparu que pour accéder à ces campagnes emblématiques, ou les modeler à l'image de leurs idéaux, les gentrifieurs convertissent effectivement leur capital économique, social, voire culturel en capital environnemental. De même qu'à l'inverse, les acteurs sociaux peuvent accumuler d'autres formes de capital par l'intermédiaire de leur capital environnemental, tendant ainsi à entretenir les logiques de domination et hiérarchies sociales en place. Il est cependant nécessaire de nuancer la portée de ces dernières, puisqu'au sein du champ environnemental se révèlent des positionnements d'acteurs parfois bien moins manichéens.

\section{Les modalités d'accès au capital environnemental}

Comme les autres, le capital environnemental peut être accumulé, investi, converti. Pour disposer du capital environnemental, les acteurs peuvent (doivent) convertir des capitaux (économique, culturel, social) dont ils disposent déjà et qu'ils choisissent d'investir dans des biens, des connaissances ou des pratiques liés à l'environnement ou fortement connotés de valeurs environnementales. De manière très prosaïque, pour s'installer dans une campagne riche en aménités, par nature plutôt environnementales, donc convoitée, les gentrifieurs potentiels doivent consentir un investissement économique parfois très lourd. En l'espèce, plus le capital environnemental escompté est élevé (habitation spacieuse, terrain vaste et paysager, cadre de vie agréable, qualités environnementales labellisées et sécurisées par des périmètres juridiques tels que les Parcs naturels, nationaux ou régionaux, les AONB 
au Royaume-Uni, les sites classés, etc.), plus il nécessitera une conversion significative de capital économique. Pour reprendre les cas d'étude, qu'il s'agisse du Montana ou des campagnes anglaises, le « ticket » d'entrée (via une résidence principale ou secondaire) est devenu on ne peut plus prohibitif, a minima très sélectif (Richard, 2017). Parfois la conversion du capital économique en capital environnemental peut d'ailleurs bénéficier à l'intérêt général. C'est le cas dans le Montana, où une partie de la population est assez fortunée pour financer la protection de l'environnement, via par exemple la Yellowstone Park Foundation, et indirectement garantir ainsi l'intégrité de son cadre de vie. Dans d'autres configurations, des individus peuvent faire le choix de sacrifier leur niveau de vie (c'est-à-dire une partie de leur capital économique) au profit de leur qualité de vie au titre de laquelle l'environnement occupe une place centrale, majorant au final leur capital environnemental : quitter un bon emploi sur la côte est ou à Paris pour un « job » harassant et peu reconnu dans le Montana ou une situation plus précaire à Millevaches pour s'épanouir dans des activités de pleine nature ou réaliser l'objectif d'une vie à très faible impact environnemental est aujourd'hui un phénomène courant. À tel point dans le Montana que le phénomène y est qualifié de «wilderness tax », le prix à payer pour vivre dans l'environnement de ses rêves.

Mais le capital économique n'est pas le seul à être converti en capital environnemental. À cet égard, les nouveaux habitants de la Montagne limousine s'appuient plutôt sur leur capital social. Ils font ainsi souvent référence au « réseau », qui peut être défini comme un tissu de relations sociales composé d'individus, d'associations, d'entreprises qui, par leurs initiatives et leurs actions, structurent le cadre de vie et instillent localement une intense vie sociale et politique. La dynamique associative ancienne et la présence de nombreux nouveaux résidents ont permis à ce réseau de croître et se densifier pour fournir à ceux qui en font partie un capital social important. Celui-ci peut être converti en capital environnemental, comme par exemple dans le cas d'une société coopérative d'intérêt collectif implantée au cœur de la Montagne limousine qui s'occupe d'habitat et d'urbanisme en milieu rural. Ses membres sont, pour la plupart, des nouveaux habitants, installés dans la Montagne depuis plus ou moins long- temps ; bien insérés dans le réseau de la Montagne, par leur engagement associatif et/ou politique, ils mobilisent et investissent leur capital social dans du capital environnemental. Leur reconnaissance et légitimité locales leur ont permis de réaliser divers projets (éco-quartier, redynamisation de centresbourgs, aménagements de lieux de vie associatifs) imprégnés de valeurs écologisantes, respectueuses de l'environnement, d'un urbanisme durable, etc.

Enfin, le cas britannique démontre qu'il est même envisageable de convertir du capital culturel en capital environnemental. En effet, l'instauration de règles d'urbanisme strictes visant à protéger les potentiels agricoles et les paysages ruraux a coïncidé avec l'intensification des flux migratoires de ménages issus des middle classes. Depuis, comme indiqué plus haut, ces dernières s'évertuent à préserver la qualité de leur environnement et de leur cadre de vie en pesant sur les déclinaisons locales des politiques d'urbanisme, d'aménagement ou encore de logement. Pour ce faire, lorsqu'ils disposent eux-mêmes de compétences, voire de qualifications professionnelles, ils s'appuient sur leur propre capital culturel. À défaut, ils peuvent sans difficulté mobiliser leurs actifs sociaux pour faire appel à des connaissances, des voisins ou autres susceptibles d'intervenir pour monter un dossier pour ou contre quoi que ce soit (de nouvelles constructions, des éoliennes, une ligne de train, cf. Bailoni, 2012), ou dialoguer très efficacement avec les autorités administratives (notamment dans les parcs nationaux) dont ils connaissent et maitrisent parfaitement les codes.

De notre point de vue, ces quelques exemples de conversion constituent autant de modalités par lesquelles les gentrifieurs acquièrent et accumulent cette forme spécifique de capital. Certes, les profils de gentrifieurs sont variés et les valeurs qu'ils mobilisent sont hétérogènes. Mais c'est justement le constat de cette diversité qui permet de valider la pertinence et la capacité opérationnelle d'une grille de lecture qui s'applique de manière transversale à des terrains différents. Le capital environnemental devient alors une clé d'interprétation pour appréhender les investissements, matériels et symboliques, des gentrifieurs, et la manière dont l'environnement devient un instrument de domination, en leur permettant de préserver leur cadre de vie, d'affirmer leur position, voire leur pouvoir sur ces territoires. 


\section{Une réversibilité de l'investissement dans le capital environnemental}

À court ou à long terme, les acteurs disposant de capital environnemental peuvent le convertir pour investir dans une autre forme d'actif. Si la conversion des différents types d'actifs vers le capital environnemental permet de saisir les moyens par lesquels les gentrifieurs accèdent à un cadre de vie privilégié, préservent sa qualité paysagère et participent à légitimer des valeurs environnementales, la réversibilité du processus contribue à mieux comprendre comment, de manière plus générale, les gentrifieurs assoient leur légitimité et affirment leur influence, le cas échéant leur emprise, sur certaines campagnes emblématiques.

En Montagne limousine, le capital environnemental peut par exemple être converti en capital économique : on pense ici aux habitants qui mettent à profit une résidence bien située, dans un cadre environnemental privilégié, pour en faire des hébergements touristiques. De même, certains gentrifieurs qui ont une connaissance fine du territoire et de ses aspects environnementaux peuvent "institutionnaliser » cette forme de capital environnemental, par exemple à travers des diplômes de guide de moyenne montagne, ou une activité d'animateur de sports de nature, et avoir un revenu directement lié à leurs connaissances et compétences sur le territoire et l'environnement.

À cet égard, la conversion peut être vue comme une étape permettant d'accumuler une autre forme d'actif, le capital symbolique : cet actif, qui rend compte de la reconnaissance et du prestige qu'un acteur a accumulé, peut être vu comme une « sublimation » des autres formes de capital (Bourdieu, 1989). Les gentrifieurs, disposant souvent de plus de capital environnemental, mais également culturel et social, parfois économique par rapport aux populations locales, ont ainsi un rôle dominant sur le territoire, ce qui leur permet d'accumuler du capital symbolique. Cela ne signifie pas que les " locaux » ne disposent pas de ces capitaux, mais qu'ils en sont souvent moins bien dotés et qu'ils peuvent ainsi se trouver dans une position de dominés ${ }^{10}$. Cela conduit à la construction et l'affirma-

10. Évoquer les gentrifieurs d'une part, les «populations locales » d'autre part, comme si ces deux groupes sociaux pouvaient se différencier de manière nette, est évidemment une approche schématique qui cache la complexité du réel. Si de nombreux travaux ont souligné les tensions tion progressives d'une identité territoriale liée aux valeurs, représentations, pratiques des gentrifieurs, et ensuite à une forme d'appropriation du territoire. Cette appropriation peut être symbolique, quand l'identité d'un groupe social est durablement associée à un espace : cela est visible dans les campagnes anglaises, espaces désormais durablement perçus comme les territoires des middle classes. De la même manière, le Montana est associé à un imaginaire de nature sauvage, mais en même temps accessible par des nouvelles élites culturelles et économiques qui en font un terrain de jeu. Enfin, la Montagne limousine est reconnue comme haut-lieu d'une ruralité alternative et militante, et les gentrifieurs, par leurs pratiques sociales et politiques, ont fait de ce territoire une sorte d'attribut identitaire. Cependant, l'appropriation symbolique s'accompagne le plus souvent d'une appropriation matérielle, qui se manifeste d'abord par les divers investissements immobiliers, mais également par une main mise (relative ou a minima localisée) sur les choix économiques, les périmètres et les actions de protection, les politiques d'aménagement ou de logement.

Ainsi, dans les contextes de campagnes gentrifiées ou en cours de gentrification, les inégalités sociales se jouent sur divers registres qu'une lecture par le prisme du capital environnemental permet de mieux décrypter. Cependant, les formes potentielles de domination, ou pour l'exprimer de manière moins manichéenne, les rapports de force entre groupes sociaux doivent être appréhendés sans a priori et avec toutes les nuances nécessaires.

voire les conflits entre ces deux catégories considérées comme antagonistes, (Bozon et Thiesse, 1986; Gilbert, 2010), elles ne sont pas homogènes : tant parmi les gentrifieurs que parmi les locaux on remarque une différenciation interne, avec des pratiques de l'environnement hétérogènes et une inégale répartition des capitaux. Ainsi, des ménages locaux partagent des valeurs et pratiques environnementales avec les nouveaux habitants, entre autres en raison de la diffusion de ces valeurs, comme souligné par Banos et Candau (2014) en Dordogne. De plus, des catégories « hybrides » brouillent encore plus cette catégorisation : des ménages revenus au territoire d'origine, des nouveaux habitants installés depuis des décennies, les enfants des gentrifieurs... Difficile de dire où s'arrête l'« ancien » et ou commence le «nouvel » habitant. L'ambigüité de cette catégorisation a à plusieurs reprises été soulignée (Cognard, 2010, Tommasi, 2014), et nous sommes conscients de la simplification qu'elle induit. Nous tenons alors à souligner que son caractère n'est pas dichotomique, mais qu'elle est retenue ici car elle apparaît efficace pour appréhender les tensions sociales que nous avons constatées sur nos terrains. D'autant plus qu'elle est convoquée par les nouveaux et anciens habitants eux-mêmes! 


\section{Des rapports de force à nuancer dans le champ de l'environnement}

L'influence des gentrifieurs sur l'environnement est, nous l'avons vu, importante. Dans certains cas ils peuvent induire ou amplifier des mutations parfois spectaculaires, par leur impact paysager par exemple (Phillips, 2005; Abrams et Gosnell, 2012; Richard et al., 2014b). Dans d'autres au contraire, parfois dans un deuxième temps et à l'issue du processus de gentrification, ils peuvent agir pour la préservation de ces mêmes paysages, ne serait-ce que pour entretenir leur idéal de ruralité (cf. par exemple M. Kondo et al., 2012 pour l'État de Washington, ou encore Little, 1987; Marsden, 1993, pour les périphéries du bassin de Londres). Dans tous les cas, les gentrifieurs s'imposent comme des acteurs (parfois) cruciaux dans les luttes de pouvoir visant à définir l'environnement local, son présent et son avenir, et justifient qu'on s'interroge sur leur positionnement dans les conflits environnementaux (Hines, 2010a; 2012), qui ne répond d'ailleurs pas toujours à leur intérêt exclusif (Abrams et al., 2013) ${ }^{11}$.

En théorie et tout en considérant les nuances déjà exprimées, dans le cadre de campagnes gentrifiées et en cours de gentrification, les rapports de force opposeraient les néo-arrivants aux anciennes populations. Les gentrifieurs disposeraient des capitaux qui leur permettent de s'implanter, d'affirmer, voire d'imposer leurs visions et pratiques environnementales. Quant aux "populations locales » ou antérieures, bien souvent moins dotées, elles se trouveraient "sans voix », disposeraient de modestes leviers d'action, ce qui conduirait à leur marginalisation, notamment vis-à-vis des enjeux environnementaux et de leur gestion. Nous pouvons en trouver un exemple dans la Montagne limousine, où cette opposition est tangible autour de la gestion de la forêt : l'industrie forestière étant une des principales filières économiques locales, la ressource en bois est objet de tensions entre, d'un côté, propriétaires forestiers et acteurs de la filière, dont une partie sont des locaux, et d'autre part, la plupart des néoarrivants qui dénoncent l'exploitation industrielle de la forêt et proposent des modes de gestion locaux (filières courtes, entreprises locales) et respectueux

11. Jesse Abrams, John Bliss et Hannah Gosnell, en valorisant l'autoréflexivité des gentrifieurs, proposent de mettre en lumière la manière dont ils peuvent justement ménager leurs effets sur les territoires d'accueil. de l'environnement. Ces derniers disposent d'outils leur permettant de faire entendre leur voix (notamment par le biais des media locaux) et d'avoir une capacité d'action (dans le cadre d'associations ou de mandats électifs) sur les pratiques d'exploitation et de gestion de la ressource.

Bien évidemment, cette opposition dichotomique est trop caricaturale pour être systématiquement opératoire, ne serait-ce que parce que selon les profils respectifs des populations locales ou des néo-arrivants, selon l'antériorité et l'intensité du processus de gentrification, l'échelle à laquelle il se décline, les frontières entre groupes sociaux sont plus ou moins nettes. L'expérience de la vallée de la Blackfoot (Montana) offre un exemple de la possibilité de collaborations entre gentrifieurs et locaux sur des thématiques environnementales : lorsque la population locale a pris conscience de la pollution de la vallée, elle s'est mobilisée pour donner naissance au Blackfoot Challenge. Sur cette terre de ranchers qui connait un processus de gentrification, de nombreux types de propriétaires - installés ici depuis plusieurs générations, néo-arrivants, organismes d'État, organisations de protection de l'environnement... - se sont pris en main de manière collective et concertée pour assurer la gestion de la vallée. Ce «community based project» a permis de trouver un consensus entre des acteurs locaux aux représentations parfois antagonistes et prônant des usages diversifiés de l'environnement, allant de l'agriculture à la foresterie en passant par la préservation et les activités récréatives. Cette initiative locale permet ainsi de concilier l'antagonisme des valeurs susceptibles d'être attachées à l'environnement et d'envisager une relation pacifiée entre gentrifieurs et population locale. De la même manière, dans le contexte de certaines campagnes anglaises gentrifiées depuis des décennies, l'entre-soi des middle classes confine à un certain exclusivisme social et conduit bien souvent à privilégier la préservation des paysages ou des espaces verts à l'implantation de nouveaux logements, a fortiori de logements abordables ou sociaux. Pour autant, il arrive que les gentrifieurs mettent leurs actifs sociaux et culturels au service des populations locales, de leurs voisins, pour leur faciliter l'accès au logement ou au foncier. Cette situation a été par exemple observée dans un hameau du Lake District dans lequel les gentrifieurs ont combiné leurs compétences (dont 
celles d'un architecte renommé) et leurs réseaux d'influence pour convaincre les autorités d'octroyer à la fille de l'agriculteur local, le droit de construire sa maison en dépit de règles d'urbanisme extrêmement strictes.

\section{Conclusion}

L'environnement apparait bien au cœur des dynamiques de gentrification d'espaces ruraux emblématiques investis par des nouvelles populations disposant dans des proportions plus ou moins substantielles de divers types de capital. Dans des contextes socio-spatiaux différents, les gentrifieurs consomment un espace naturel, selon leurs orientations et valeurs en matière d'habitat, de pratiques, d'engagements, etc. Si cette approche valide les arguments pour une gentrification « du côté de la consommation », elle n'exclut pas pour autant les arguments "du côté de la production » : en effet, les modifications apportées par les gentrifieurs à l'environnement, de l'échelle domestique à l'échelle locale ou régionale, participent voire déterminent l'accueil de nouveaux gentrifieurs, qui trouvent les meilleures conditions possibles afin de satisfaire ou incarner les valeurs environnementales auxquelles ils adhèrent, complétant leur portefeuille d'actifs liés au capital environnemental.

De même, de par la construction même des deux concepts, gentrification et capitaux (économiques, sociaux, culturels...) sont par nature articulés, permettant ainsi d'expliquer les inégalités inhérentes au processus. De sorte qu'en considérant la relation des gentrifieurs à l'environnement, nous avons été conduits à esquisser et proposer la grille d'analyse du capital environnemental dont nous nous sommes efforcés de montrer qu'elle était très opérationnelle pour interpréter les divers composants et mécanismes à l'œuvre dans le processus de gentrification.

Cet actif social permet de rendre compte des valeurs mobilisées par les gentrifieurs et des investissements réalisés en leur nom, au sein d'espaces de nature emblématiques. Il s'est par ailleurs révélé très efficace pour faire ici une lecture beaucoup plus transnationale que comparative de la gentrification, des pratiques et représentations des divers types de gentrifieurs rencontrés, ainsi que des rapports de force entre groupes et acteurs sociaux. Le capital environnemental met en effet en lumière les inégali- tés, voire les injustices, liées à l'environnement, ainsi que l'existence de «dominés » et de «dominants». Les premiers sont alors susceptibles d'être physiquement exclus du territoire lorsqu'il leur est impossible d'y accéder financièrement, parfois d'être relégués au sein des portions les moins désirables des campagnes, ou enfin d'être symboliquement « effacés » des territoires dont l'identité est désormais fondée sur de nouvelles valeurs. C'est par ailleurs par les «dominés » que les travaux sur la justice environnementale sont généralement abordés. Or, l'application de la grille de lecture capital environnemental permet d'appréhender ces inégalités du côté des "dominants », ceux qui, par leurs actions, leurs (non) choix, leurs discours ou leurs silences, peuvent tendre soit à les réduire, soit à les aggraver.

\section{Bibliographie}

Abrams J.B., Bliss J., Gosnell H., 2013. Reflexive Gentrification of Working Lands in the American West: Contesting the "Middle Landscape", Journal of Rural and Community Development vol. 8, n 3, p. 144-158.

Abrams J. B., Gosnell H., 2012. The Politics of Marginality in Wallowa County, Oregon: Contesting the Production of Landscapes of Consumption, Journal of Rural Studies, vol. 28, nº 1, p. 30-37.

Bailoni M., 2012. Aménager un espace idéalisé : identité et conflits dans la campagne anglaise, Revue Géographique de l'Est (en ligne), vol. 45, no 3-4, URL: [http://rge.revues. $\mathrm{org} / 3739]$.

Banos V., Candau J., 2014. Sociabilités rurales à l'épreuve de la diversité sociale, Versailles, Quæ, 252 p.

Berque A., 2010, Histoire de l'habitat idéal: De l'Orient vers l'Occident, Paris, Éd. du Félin, 396 p.

Blanc N., 2008. Éthique et esthétique de l'environnement, EspacesTemps.net, 31.01.2008, [http://www.espacestemps. net/articles/Ethique-et-esthetique-de-environnement/].

Bourdieu P., 1989. La noblesse d'État? Grandes écoles et esprit de corps, Paris, Minuit, $576 \mathrm{p}$.

Bourdieu P., 2000. Propos sur le champ politique, Presses universitaires de Lyon, $112 \mathrm{p}$.

Bozon M., Thiesse A.-M., 1986. La terre promue. Gens du pays et nouveaux habitants dans les villages $d u$ Valois, Asnière-surOise, Fondation Royaumont, 206 p.

Bryson J., Wyckoff W., 2010. Rural gentrification and nature in the Old and New Wests, Journal of Cultural Geography, vol. $27, \mathrm{n}^{\circ} 1$, p. 53-75.

Bunce M., 1994. The Countryside Ideal: Anglo-American Images of Landscape, London, Routledge, 256 p.

Cailly L., 2007. Capital spatial, stratégies résidentielles et processus d'individualisation, Annales de géographie n 654(2), p. 169-187. 
Charmes E., 2011. La ville émiettée: essai sur la clubbisation de la vie urbaine, Paris, PUF, 296 p.

Cloke P., 1979. Key settlement in rural areas, London/New York, Methuen \& Co., 259 p.

Cloke P., 1983. An introduction to rural settlement planning, London, New York, Methuen \& Co., 380 p.

Cloke P., Phillips M., Thrift N., 1995. The new middle classes and the social constructs of rural living in BUTLER T., SAvage M. (dir.), Social change and the middle classes, London, UCL Press, p. 220-240.

Cloke P., Phillips M., Thrift N., 1998. Class colonisation and lifestyle strategies in Gower in Boyle P., Halfacree K. (dir.), Migration into rural areas: theories and issues, Chichester, Wiley, p. 166-185.

Cloke P., Thrift N., 1987. Intra-class Conflict in Rural Areas, Journal of Rural Studies vol. 3, n 4, p. 321-333.

Cognard F., 2006. Le rôle des recompositions sociodémographiques dans les nouvelles dynamiques rurales: l'exemple du Diois. Méditerranée [Online], nº 107.

Cognard F., 2010. «Migrations d'agrément » et nouveaux habitants dans les moyennes montagnes françaises : de la recomposition sociale au développement territorial. L'exemple du Diois, du Morvan et du Séronais, Thèse de doctorat en géographie, Université Blaise-Pascal, Clermont-Ferrand, 528 p.

Darling E.J., 2005. The City and the Country: Wilderness Gentrification and the Rent Gap, Environment and Planning vol. 37, p. 1015-1032.

Desponds D., 2007. Les impacts d'un parc naturel régional (PNR) sur les évolutions socio-démographiques de son espace rural: le cas du Vexin français, Norois. Environnement, aménagement, société $\mathrm{n}^{\circ} 202$, p. 47-60.

Friedberger M., 1996. Rural Gentrification and Livestock Raising: Texas as a Test Case, 1940 1995, Rural History, vol. $7, \mathrm{n}^{\circ} 1$, p. 53-68.

Ghose R., 2004. Big sky or big sprawl? Rural gentrification and the changing cultural landscape of Missoula, Montana, Urban geography 25(6), p. 528-549.

Gilbert Y., 2010. Migrations urbaines et milieu rural : diversification sociale et recomposition du politique, Espaces et sociétés, vol. 3, nº 143, p. 135-159.

GLAss R., 1964. Introduction in Centre for Urban Studies (Ed.), London, Aspects of Change, London, Macgibon \& Kee, pages XIII-XLII.

Gosnell H., Travis W.R., 2005. Ranchland Ownership Dynamics in the Rocky Mountain West, Rangeland Ecology \& Management, vol. 58, n² 2, p. 191-198.

Halfacree K., 2011 , Radical Spaces of Rural Gentrification, Planning Theory E Practice 12(4), p. 618-625.

Hamnett C., 1997. Les aveugles et l'éléphant : l'explication de la gentrification, Strates [En ligne], nº 9, 1997. URL: [http://strates.revues.org/611].

Hines J.D., 2007. The persistent frontier and the rural gentrification of the Rocky Mountain West, Journal of the West vol. 46, no 1, p. 63-73.

Hines J.D., 2010. Rural gentrification as permanent tourism : the creation of the New West Archipelago as postindus- trial cultural space, Environment and Planning: Society and Space vol. 28, p. 509-525.

Hines J.D., 2012. The Post-Industrial Regime of Production/ Consumption and the Rural Gentrification of the New West Archipelago, Antipode, vol. 44, n 1, p. 74-97.

Hoggart K., 1997. The middle classes in rural England, 19711991, Journal of Rural Studies, vol. 13, n 3, p. 253-273.

Horton J., 2008. Producing Postman Pat: the popular cultural construction of idyllic rurality, Journal of Rural Studies. vol. 24, no 4, p. 389-398.

Keucheyan R., 2014. La nature est un champ de bataille, Paris, La Découverte, 176 p.

Kondo M., Rivera R., Rullman S., 2012. Protecting the idyll but not the environment: Second homes, amenity migration and rural exclusion in Washington State, Landscape and Urban Planning $\mathrm{n}^{\circ}$ 106, p. 174-182.

LeVy J., 2003. Capital Spatial, in LÉvy J., Lussault M. (éds.), Dictionnaire de la géographie et de l'espace des sociétés, Paris, Belin.

LEY D., 1980. Liberal ideology and post industrial city. Annals of the Association of American Geographers, vol. 70, p. 238-258.

LitTLE J., 1987. Rural gentrification and the influence of locallevel planning in CLOKE P. (dir.), Rural planning: policy into action?, London, Harper \& Row, p. 185-199.

Marsden T., Murdoch J., Lowe P., Munton C.R, Flynn A., 1993. Constructing the countryside, Westview Press, $232 \mathrm{p}$.

Mathis C.F., 2010. In Nature we trust. Les paysages anglais à l'ère industrielle, Paris, Presses de l'université de Paris-Sorbonne, $688 \mathrm{p}$.

McLean N., 1976. A river runs through it and Other Stories, Chicago, University of Chicago Press 231 p.

Neveu É., 2013. Les sciences sociales doivent-elles accumuler les capitaux ?, Revue française de science politique, vol. 63, $\mathrm{n}^{\circ} 2$, p. 337-358.

Newby H., 1979. Green and pleasant land? Social change in rural England, London, Hutchinson of London, 301 p.

PAHL R. E., 1965. Urbs in rure: the metropolitan fringe in Hertfordshire, London, London School of Economics and Political Science, $92 \mathrm{p}$.

Parsons D., 1979. A geographical examination of the twentieth century theory and practice of selected village development in England, PhD Thesis, University of Nottingham.

PARSONS D., 1980. Rural gentrification: the influence of rural settlement planning policies. Brighton, University of Sussex, $36 \mathrm{p}$.

Perrenoud M., 2008. Les artisans de la «gentrification rurale » : trois manières d'être maçon dans les Hautes-Corbières, Sociétés contemporaines, vol. 71, nº 3, p. 95-115.

Phillips M., 1993. Rural gentrification and the processes of class colonisation, Journal of Rural Studies, vol. 9, $\mathrm{n}^{\circ} 2$, p. 123-140.

Phillips M., 2002. The production, symbolization and socialization of gentrification: impressions from two Berkshire villages, Transactions of the Institute of British Geographers, vol. 27, n 3, p. 282-308.

Phillips M., 2005. Rural gentrification and the production of nature: a case study from Middle England, in Ramirez, B. 
(dir.), Papers from the $4^{\text {th }}$ International Conference of Critical Geographers, Mexico City.

Phillips M., 2007. Changing class complexions on and in the British countryside, Journal of Rural Studies, vol. 23, p. 283304.

Pistre P., 2012. Renowveaux des campagnes françaises : évolutions démographiques, dynamiques spatiales et recompositions sociales, Thèse de doctorat, Université Paris-Diderot - Paris 7, $420 \mathrm{p}$.

Raymond S., 2005. Des risques de marginalisation aux risques de gentrification : espaces ruraux convoités et inégalement accessibles, in Arlaud S., Jean Y., Royoux D. (dir.), RuralUrbain, nouveaux liens, nouvelles frontières, Rennes, PUR, p. 235-249.

RedFond R., 1992. A river runs through it, [DVD], EberTs J., Redford R., Markey P., Columbia Pictures, $123 \mathrm{~min}$.

Richard F., 2009. La gentrification des «espaces naturels» en Angleterre : après le front écologique, l'occupation?, L'Espace Politique [En ligne], 9 | 2009-3, URL : [http:// espacepolitique.revues.org/1478].

RichaRD F., 2017. La gentrification rurale, de l'observation du fait géographique à la circulation du concept, Habilitation à diriger des recherces, vol. 1, Université de Limoges, 227 p.

Richard F., Chevallier M., Dellier J., Lagarde V., 2014 a. Circuits courts agroalimentaires de proximité en Limousin : performance économique et processus de gentrification rurale; Norois. Environnement, aménagement, société, no 230, p. 21-39.

Richard F., Dellier J., Tommasi G., 2014b. Migration, environnement et gentrification rurale en Montagne limousine, Journal of Alpine Research | Revue de géographie alpine, $\mathrm{n}^{\circ}$ 102-104.

Richard F., Saumon G., Tommasi G., 2015. De l'hégémonie des valeurs environnementales à l'affirmation d'un nouveau capital?, Colloque Capital environnemental : pra- tiques, dominations, représentations, appropriations spatiales, Limoges, 18-21 novembre 2015.

Ripoll F., Veschambre V., 2005, Sur la dimension spatiale des inégalités : contribution aux débats sur la «mobilité » et le « capital spatial », in Arlaud S., Jean Y., Royoux D. (dir.), Rural - Urbain, Nouveaux liens, nowvelles frontières, Rennes, PUR, p. 467-483.

SAumon G., 2016. Du personnage du cow-boy solitaire à celui du pêcheur à la mouche : la fiction au fondement de la mutation territoriale de l'ouest du Montana?, Annales de géographie, no 709-710, p. 309-332.

Sмiтн D.P., 1998. The revitalisation of the Hebden Bridge District: gentrified Pennine rurality, $\mathrm{PhD}$ Thesis, University of Leeds.

Sмiтн D.P., 2002a. Rural gatekeepers and "greentrified" pennine rurality: Opening and closing the access gates?, Social E Cultural Geography, vol. 3, n 4, p. 447-463.

Smith D.P., Phillips D.A., 2001. Socio-cultural representations of greentrified Pennine rurality, Journal of Rural Studies, vol. $17, \mathrm{n}^{\circ} 4$, p. 457-469.

Sмiтн N., 1987. Gentrification and the rent gap, Annals of the American Association of Geographers, vol. 77, p. 462-478.

Sutherland L.A., 2012. Return of the Gentleman Farmer? Conceptualising Gentrification in UK Agriculture, Journal of Rural Studies, vol. 28, n 4, p. 568-576.

Tommasi G., 2014. Vivre (dans) des campagnes plurielles. Mobilités et territoires dans les espaces ruraux. L'exemple de la Sierra de Albarracin et du Limousin, Thèse de doctorat, Université de Limoges, 457 p.

Travis W., 2007. New Geographies of the American West, Washington, Island Press, 304 p.

Woods M., 2016. Reconfigurating places - wealth and the transformation of rural areas, in Hay I., Beaverstock J.V. (dir.), Handbook on Wealth and the Super-Rich, Cheltenham UK/Northampton USA, Edward Elgar, p. 264-286. 